À paraître dans S. Clément-Tarantino - F. Klein (dir.), La représentation du 'couple' Virgile-Ovide dans la culture, de l'Antiquité à nos jours, Presses du Septentrion, Villeneuve-d'Ascq.

\title{
Le Fromage et le Dentifrice : le couple Virgile-Ovide dans les Xenia et les Apophoreta de Martial
}

\author{
Évelyne Prioux*
}

Bien que la présentation que Martial donne d'Ovide soit loin d'être univoque, Ovide est le poète que Martial cite le plus volontiers si nous nous fions, du moins, aux effets d'intertextualité relevés par de nombreux commentateurs à la suite de l'étude proposée par Zingerle en $1877^{1}$. Ce constat peut paraître d'autant plus étonnant et paradoxal que Martial cite très peu Ovide par son nom ou de manière explicite, contrairement à ce que l'on peut constater au sujet de ses allusions à Catulle. S. Hinds a récemment montré l'intérêt qu'il pouvait y avoir, pour mieux cerner le positionnement

ㅍ CNRS / Université de Paris Ouest-Nanterre-La Défense (UMR 7041 ArScAn, équipe ESPRI). Les recherches relatives au présent dossier ont été effectuées dans le cadre du programme d'ANR CAIM (Culture antiquaire et invention de la modernité).

Zingerle A., Martial's Ovid-Studien, Innsbruck, Wagner'sche Universitäts-Buchhandlung, 1977 ; Siedschlag E., « Ovidisches bei Martial », Rivista di Filologia e di Istruzione Classica, n 100, 1972, p. 156-161 ; Sullivan J. P., Martial, the Unexpected Classic : A Literary and Historical Study, Cambridge, Cambridge University Press, 1991, p. 105-107 ; Connors C., "Seeing Cypresses in Virgil », The Classical Journal, n 88-1, 1992, p. 1-17, spéc. 4-12 ; Pitcher R. A., « Martial's debt to Ovid », dans F. Grewing (dir.), Toto Notus in Orbe : Perspektiven der Martial-Interpretation, Stuttgart, Franz Steiner Verlag, 1998, p. 59-76 ; Szelest H., « Ovid und Martial », dans W. Schubert (dir.), Ovid : Werk und Wirkung. Festgabe für Michael von Albrecht zum 65. Geburtstag, Frankfurt-am-Main, Peter Lang, 1999, t. 2, p. 861-864; Coleman K., Martial : Liber Spectaculorum. With introduction, translation and commentary, Oxford: Oxford University Press, 2006, p. 117-118, 176, 200, 207, 247 ; Hinds S., « Martial's Ovid / Ovid's Martial », The Journal of Roman Studies, n 97, 2007, p. 113-154 ; Deremetz A., « Virgile et Ovide dans les Épigrammes de Martial », dans le présent volume. 
de Martial par rapport à la figure d'Ovide, à se focaliser sur les trois premiers recueils publiés par Martial : le Livre des spectacles, les Xenia et les Apophoreta, trois œuvres où la définition de l'identité poétique de Martial apparaît comme un enjeu majeur². C'est sur ces mêmes recueils, et plus particulièrement sur les Xenia et les Apophoreta, que je me propose de revenir pour examiner comment Martial représente le « couple » Virgile-Ovide.

\section{Le projet poétique des Apophoreta et des Xenia et ses modèles}

Avant d'aller plus loin, j'aimerais souligner que, dans l'œuvre de Martial, le « couple » Virgile-Ovide ne peut guère être appréhendé en lui-même : premièrement, si l'on s'en tient à l'examen des effets d'intertextualité, Martial imite bien plus souvent Ovide que Virgile ${ }^{3}$. Deuxièmement, Virgile et Ovide font partie d'une nébuleuse de modèles que Martial convoque à tour de rôle et ne sont qu'exceptionnellement isolés comme couple ou directement confrontés (le contre-exemple le plus saillant est l'épigramme sur le cerf, gibier offert pour la consommation en XIII, 96, et qui est successivement comparé à l'animal de Cyparissus connu par les Métamorphoses d'Ovide et à celui de Silvia dans l'Énéide ${ }^{4}$ ). S'il s'agit d'identifier les modèles latins de Martial, le projet des Xenia et des Apophoreta nous conduira plus naturellement à tourner nos regards non vers un « couple » Virgile-Ovide, mais vers le couple Catulle-Ovide. De fait, on retrouvera de fréquentes mentions de Catulle, qui le confrontent souvent à d'autres modèles possibles, dont Virgile et Ovide. En XIV, 195, Martial s'intéresse ainsi au couple Virgile-Catulle, et annonce que la grande Vérone doit autant à Catulle que la petite Mantoue à Virgile $^{5}$ : dans cette épigramme, Martial place son principal prédécesseur dans le genre épigrammatique au même niveau ou même au-dessus de celui qui était reconnu, par ses contemporains, comme le plus grand des poètes épiques latins. Ce poème me paraît particulièrement intéressant car il évoque immanquablement deux passages où Ovide se situe par rapport à ses prédécesseurs : Remèdes à l'amour, 395-396, où Ovide établit un parallèle entre le rôle de Virgile dans le genre épique et son propre rôle dans le genre élégiaque ${ }^{6}$, et Amours III, 15, dernière pièce du recueil des Amours, où Ovide compare Virgile, gloire de Mantoue, à Catulle, fierté de Vérone, et se présente comme étant lui-même l'honneur du peuple pélignien, qui fera, par sa renommée, de la minuscule Sulmone une grande ville. La même pièce annonce que le poète quittera désormais les thèmes érotiques pour se tourner vers le thyrse plus lourd du puissant Bacchus $^{7}$. Lorsque Martial emprunte les propres mots d'Ovide, mais avec l'intention de resserrer

\footnotetext{
$2 \quad$ Hinds S., Ibid.

3 Sur la place de l'hypotexte virgilien chez Martial, voir Spaeth Jr., J. W., « Martial and Vergil », Transactions and Proceedings of the American Philological Association, ${ }^{\circ}$ 61, 1930, p. 19-28 ; Sullivan J. P., op. cit., p. 102-103 ; Connors C., art. cit., spéc. 4-12. Les jugements de Martial sur Virgile sont notamment commentés par Preston K., « Martial and Formal Literary Criticism », Classical Philology n 15, 1920, p. 340352.

Connors C., art. cit., spéc. 4-12.

Martial, Épigrammes, XIV, 195 : Catullus / Tantum magna suo debet Verona Catullo, / Quantum parua suo Mantua Vergilio. («Catulle. La grande Vérone doit autant à son cher Catulle que la petite Mantoue à son cher Virgile »).

Ovide, Remèdes à l'amour, 395-396 : Tantum se nobis elegi debere fatentur / quantum Vergilio nobile debet epos (« Les vers élégiaques avouent qu'ils nous doivent autant que la noble épopée doit à Virgile »).
} 
le canon poétique qu'il présente autour des deux seules figures de Catulle et de Virgile, on peut légitimement soupçonner un coup de griffe malicieux à l'endroit du poète de Sulmone ${ }^{8}$.

Par son projet même, le Martial des Apophoreta nous invite cependant à regarder vers un « couple » Catulle-Ovide, deux poètes avec l'œuvre desquels Martial ne cessera de dialoguer de manière privilégiée. C'est en effet chez ces deux poètes que l'on trouvera les modèles les plus proches du projet de Martial, modèles qu'il tourne volontiers en dérision. Les Xenia (présents d'hospitalité destinés à être mangés) et les Apophoreta (cadeaux divers) se présentent, dans leurs pièces liminaires, comme des recueils étroitement associés aux réjouissances des Saturnales. Le premier recueil se compose de distiques précédés d'un titre qui décrivent des victuailles, qui, pour certaines, sont prêtes à être consommées et, pour d'autres, requièrent une préparation culinaire ${ }^{9}$. Le second décrit des présents, des lots qui peuvent tirés au sort et qui sont disposés par paires faisant alterner un présent destiné à un riche avec un présent destiné à un pauvre ${ }^{10}$.

Ces livres sont explicitement associés à la célébration des Saturnales et la dernière pièce des Apophoreta, qui concerne des beignets (adipata) donnés aux écoliers qui reprennent le chemin de l'école une fois les Saturnales passées, suppose que la lecture du livre s'achève précisément au moment où finit la fête ${ }^{11}$. Le fait d'envoyer un livre à l'occasion des Saturnales nous renvoie naturellement au Carmen 14 de Catulle qui reproche à son ami Calvus de lui avoir envoyé (sans doute par jeu ?) une anthologie poétique rassemblant l'arrière ban des poètes de leur temps et de vouloir ainsi le faire mourir dès le lendemain des Saturnales. Dans le recueil de Catulle, cette épigramme s'intègre dans une courte série de carmina (12-14) qui concernent les présents entre amis ${ }^{12}$. Il semble évident que ce modèle du livret exécrable était présent à l'esprit de Martial puisque les Apophoreta contiennent précisément une série d'épigrammes sur des livres offerts en cadeaux qui s'achève précisément sur la présentation des Carmina de Catulle et d'un traité de Calvus ${ }^{13}$, présenté par Martial comme un livre exécrable et donc un apophoreton aussi mauvais que celui reçu par Catulle dans le Carmen 14.

7 Ovide, Amours, III, 15, 7-8 : Mantua Vergilio, gaudet Verona Catullo ; / Paelignae dicar gloria gentis ego, ( «Mantoue est fière de Virgile, et Vérone de Catulle ; on m'appellera, moi, l'honneur du peuple pélignien »). Sur cette épigramme qui sert également de modèle à Martial, Ép., I, 61 voir, ici-même, l'article d'A. Deremetz.

8

Martial, Ép., VIII, 73, 9 qui s'inspire encore du modèle d'Amours III, 15 détache au contraire clairement un couple Virgile-Ovide (renforcé par l'évocation conjointe d'Alexis et de Corinne) au sein d'un catalogue de poètes connus pour leurs amours.

En ceci, les Xenia de Martial, tout comme les motifs ornant les parois de IV $^{\mathrm{e}}$ style pompéien ou le second des deux tableaux de xenia décrits par Philostrate l'Ancien à la fin de ses livres d'Eikones, s'écartent de la définition stricte du genre pictural des xenia (représentation de victuailles prêtes à être consommées) qui est donnée par Vitruve (De l'architecture, VI, 7, 4). Voir Bryson N., Looking at the Overlooked. Four Essays on Still Life Painting, Londres, Reaktion books, 1990, chap. 1.

Martial, Épigrammes, XIV, 1, 5.

Pour cette remarque, voir Citroni M., «Marziale e la letteratura per i Saturnali (poetica dell' intrattenimento e cronologia della pubblicazione dei libri)», Illinois Classical Studies, n 14, 1989, p. 201226, spéc. 210-211.

12 Voir Young Forsyth P., « Gifts and Giving : Catullus 12-14 », The Classical World, n 78-6, 1985, p. 571574.

Voir le couple formé par les épigrammes XIV, 195 et 196 (livre de Catulle et traité De aquae frigidae usu de Calvus) au sein de la série de distiques accompagnant des livres offerts en cadeau (XIV, 183-196). 
Le motif du livre composé à l'occasion des Saturnales sera par ailleurs repris par Ovide dans les Tristes. En Tristes II, 471-491, Ovide exilé souligne qu'il n'est pas le premier à avoir écrit un traité concernant une activité frivole et mentionne l'existence de traités sur les jeux de hasard, sur la natation, sur l'art du maquillage, sur les règles du banquet et de l'hospitalité, sur les types d'argile servant à modeler les coupes et sur les types d'amphores adaptées à la conservation du vin. Cette littérature didactique consacrée à des sujets frivoles correspondrait à une tradition bien établie dans le cadre des Saturnales : Talia luduntur fumonso Decembri, dit Ovide (Tr. II, 491). Martial, qui imitera la formule d'Ovide dans l'Épigramme V, 30 pour opposer sa propre poésie qui convient à la « fumée de Décembre » (fumoso [...] Decembri) à l'« élégie à la coiffure soignée » (cultis [...] Elegia comis) $)^{14}$, joue sur le modèle d'une littérature didactique centrée sur la fête et le banquet ${ }^{15}$ : si ses recueils de monodistiques n'ont pas une forme didactique, ils livrent cependant des recettes pour utiliser les victuailles des Xenia, donnent des conseils sur l'utilisation des différents présents et comparent la qualité et la dignité des produits en fonction de leur origine géographique. Il est également intéressant de noter que le passage des Tristes qui vient d'être mentionné insiste notamment sur le fait qu'Ovide, lui, n'a pas écrit de traité sur les jeux de hasard, alors que ces activités autrefois prohibées fournissaient le sujet d'ouvrages écrits par d'autres auteurs et de comparer les vers où Ovide inventorie les jeux dont il s'est vertueusement abstenu de parler dans ses poèmes didactiques ( $T r$. II, 471-484) à l'intérêt que les poèmes liminaires du livre des Apophoreta de Martial portent aux jeux de hasard et au matériel de jeu qui leur est associé (XIV, 14-19). Or, Martial, qui choisit de s'attacher à ces jeux et même de leur donner une place de choix dans la définition de sa poétique ${ }^{16}$, se plaît à pointer sa source et à mettre en évidence, par un effet d'intertextualité, la manière dont il subvertit le modèle ovidien : A. Zingerle ${ }^{17}$ note ainsi la double source du pentamètre de l'épigramme XIV, 17 qui porte sur une table de jeu réversible, dont l'une des faces peut accueillir le ludus scriptorum et où je serais tentée de reconnaître une image réflexive du jeu poétique de Martial. La formule calculus hac gemino discolor hoste perit évoque en effet Tr. II, 477-478 (discolor ut recto grassetur limite miles, / cum medius gemino calculus hoste perit), mais aussi le passage de l'Art d'aimer qui compare les talents de l'élève d'Ovide en matière d'amour à ceux d'un habile joueur de dés $(A$. $A$., III, 358 : unus cum gemino calculus hoste perit)! Le projet poétique des Apophoreta se constitue ainsi autour d'une inversion revendiquée du modèle ovidien. Au lusus érotique pris comme sujet d'une élégie didactique succède le choix pleinement assumé d'un sujet qu'Ovide laissait à d'autres : les jeux de hasard, que Martial ira jusqu'à ériger en métaphore possible de l'écriture poétique.

Le modèle de la poésie didactique ovidienne est lui aussi repris par Martial, même s'il est constamment tourné en dérision. Il paraît en effet légitime de supposer que les Medicamina faciei femineae étaient particulièrement présents à l'esprit de l'épigrammatiste lorsque celui-ci composa des épigrammes destinées à accompagner des cadeaux d'ordre cosmétique (XIV, 24-27 et 56-60).

\footnotetext{
14

Martial, Ép., V, 30, 5 : fumoso [...] Decembri.

15 Sur ce point, voir Citroni M., 1989, art. cit.

16 Voir Roman L., « A History of Lost Tablets », Classical Antiquity, n 25-2, 2006, p. 351-388, spéc. p. 381, où l'auteur note que Martial substitue à l'intérêt d'Ovide pour le lusus pris dans un double sens érotique et poétique un intérêt pour le lusus pris au sens de jeu de hasard et de jeu poétique.

Zingerle A., op. cit., p. 20.
} 
Ainsi pour le dentifrice offert en XIV, 56, qui prend la parole pour s'indigner d'être offert non à une puella, mais au propriétaire d'un dentier ${ }^{18}$ : il est intéressant de noter que le rôle du dentifrice est ici de polire, "polir », un terme qui a une longue histoire dans le registre métapoétique, tout comme la puella qui symbolise l'élégie et une forme poétique bien éloignée des épigrammes satiriques de Martial. L'irruption de la puella et du poli ou de la leuitas des cosmétiques chers à Ovide ${ }^{19}$ dans un univers qui leur est étranger est ici soulignée par l'indignation du dentifrice qui parle à la première personne : quid mecum est tibi ? Le changement de locuteur qui démarque cette épigramme de celles qui l'entourent immédiatement doit peut-être suggérer que le tibi visé par le dentifrice pourrait se confondre avec Martial lui-même. On peut en outre noter que la formule quid mecum est tibi évoque plusieurs hypotextes ovidiens, comme si la voix prêtée au dentifrice se superposait avec le souvenir des vers d'Ovide ${ }^{20}$.

À supposer que le dentifrice de XIV, 56 ait quelque chose d'ovidien, il serait intéressant de noter que l'épigramme suivante met en évidence l'étrangeté qu'aurait eu un autre produit cosmétique, le myrobalanum (myrobalan ou noix de Ben), pour Virgile ${ }^{21}$. On ignore si Ovide avait mentionné ce produit, et, si c'était le cas, par quel tour périphrastique il l'avait fait (Martial y parvient en remplaçant myron par unguentum). Le terme était sans doute associé assez étroitement à la culture alexandrine dans la mesure où le myrobalan provenait notamment de la côte trogodytique ${ }^{22}$ comme de nombreux autres produits cosmétiques dont l'exploitation pourrait s'être développée sous l'impulsion des premières reines lagides ${ }^{23}$. Qu'Homère et que Virgile n'aient pu, comme le souligne Martial, employer ce terme s'explique bien sûr par le fait qu'une telle séquence de quatre syllabes brèves ne puisse entrer dans un hexamètre dactylique. L'intérêt

18 Martial, Ép., XIV, 56 : Dentifricium / Quid mecum est tibi ? Me puella sumat : / emptos non soleo polire dentes. (« Dentifrice. Qu'ai-je à faire avec toi ? C'est à une jeune fille de me recevoir : je n'ai pas coutume de polir des dents d'emprunt. »)

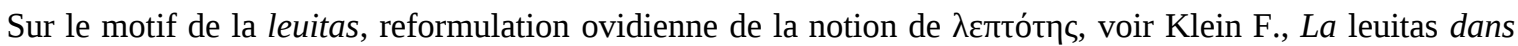
l'œuvre ovidienne. Étude d'une catégorie poétique dans le système littéraire de la Rome augustéenne, thèse de doctorat de l'Université Lille 3 - Charles-de-Gaulle, 2008. Sur l'aspect programmatique et métapoétique de l'intérêt ovidien pour les cosmétiques et pour l'art de se parer, voir Heldmann K., « Ovid Über den Pfau : Zum Lobe der Schönheit », Hermes, n 110, 1982, p. 375-380 ; Watson P. A., "Ovid and Cultus : Ars Amatoria 3.113-328 », Transactions of the American Philological Association, n 112, 1982, p. 237-244 ; Rosati G., Ovidio. I cosmetici delle donne, Venise, Marsilio, 1985, introduction ; Watson P. A., « Parody and Subversion in Ovid's Medicamina faciei femineae ", Mnemosyne, $4^{\mathrm{e}}$ série, $n^{\circ}$ 54, 2001, p. 457-471; Prioux É., " Ovide, De Medicamine faciei », dans I. Bardiès-Fronty - Ph. Walter - M. Bimbenet Privat (dir.), Le Bain et le Miroir. Catalogue de l'exposition du Musée National du Moyen Âge et du Musée National de la Renaissance (maiseptembre 2009), Paris, Gallimard, 2009, p. 231-233.

20 Cf. Ovide, Amours, III, 6, 87 (Quid mecum, furiose, tibi ?, dans contexte programmatique de l'adresse à un fleuve en crue) ou encore l'incipit de Tristes, II, 1 (Quid mihi uobiscum est, infelix cura, libelli, vers auquel Martial répond malicieusement en Ép. II, 22, 1 : voir Hinds S., art. cit., p. 119). Je remercie F. Klein et J.-Chr. Jolivet d'avoir attiré mon attention sur l'éventuelle résonance ovidienne de la formulation s occurrences de quid mecum est tibi.

Martial, Ép., XIV, 57 : Myrobalanum / Quod nec Vergilius nec carmine dicit Homerus, / Hoc ex unguento constat et ex balano. («Myrobalan. Ce nom qu'on ne trouve ni chez Virgile ni chez Homère est composé à partir du parfum et du gland. »).

Pline l'Ancien, Histoire naturelle, XII, 100.

Pour l'hypothèse selon laquelle l'exploitation des matières cosmétiques issues de la côte trogodytique aurait été liée à l'intérêt des premières reines lagides pour la parfumerie, voir Jackson S., "Callimachus : Coma Berenices : Origins », Mnemosyne, 4e série, nº 54-1, 2001, p. 1-9, spéc. p. 3-4. 
pour le nom du myrobalan, qui désigne à la fois le produit cosmétique qui en est tiré (myron) et le nom du fruit dont il est extrait (balanos) se retrouve chez Pline ${ }^{24}$, mais on peut supposer que Martial s'amuse ici à dévoyer ce produit en mettant en valeur le terme de balanos, qui peut aussi bien désigner un fruit que le gland de la verge ${ }^{25}$ et que ce sous-entendu était d'autant plus perceptible qu'il détache dans l'épigramme suivante (XIV, 58) les composants du terme aphronitre (aphros, écume associée de manière bien connue au sperme, et nitron). Doit-on aller jusqu'à penser que Martial établit, dans l'épigramme sur le myrobalan, un partage entre la poésie de Virgile, l'Homère latin, qui n'aurait pas employé ce terme (non seulement pour des raisons métriques, mais aussi parce que le sujet était très éloigné des préoccupations virgiliennes), et celle de l'expert en cosmétiques qu'était Ovide, dont l'alexandrinisme raffiné nous ramènerait en réalité au pénis et dont Martial se plairait à souligner le crimen ? Quoi qu'il en soit, la lecture demeure incertaine en raison de l'auto-dérision de Martial qui, lui, parvient précisément à nommer le myrobalan dans son poème et est généralement friand de toute possibilité d'allusion à un phallus.

L'art de dévoyer la présentation des cosmétiques est lié, chez Martial, à la présentation satirique de leurs destinataires : à l'instar du dentifrice initialement adressé à un individu pourvu d'un dentier, une teinture à cheveux est proposée à une vieille femme menacée par la calvitie ${ }^{26}$. Ce procédé consistant à détourner les conseils cosmétiques et les éventuels souvenirs des élégies didactiques d'Ovide est repris en II, 41 où Martial feint de citer Ovide pour dire que ses conseils sur le rire ne sauraient être adressés à Maximina qui n'a plus que trois dents couleur de buis et de poix. L'effet d'intertextualité est d'autant plus complexe que la citation est modifiée et que Martial s'inspire d'une section de l'Art d'aimer (3.279-282) où il s'agit, en réalité, de savoir s'accommoder des défauts physiques et où, avant de donner des conseils aux filles sur la manière de rire, Ovide intégrait, parmi ses auditrices, celles qui avaient une dent noire, trop longue ou mal placée et leur indiquait, tout comme le fait Martial, qu'elles se faisaient beaucoup de tort en riant ${ }^{27}$. Comme le souligne S. Hinds le passage auquel Martial s'est ici attaché est en réalité l'un de ceux où la poétique d'Ovide préfigure peut-être le plus celle de Martial, mais Martial se plaît, tout en imitant Ovide, à dévoyer l'image de la puella et des «douces jeunes filles ${ }^{28}$, qui font partie des images programmatiques du genre élégiaque, pour leur substituer de risibles édentées, qui sont peut-être les métaphores grimaçantes de ses propres choix esthétiques et de sa « poétique du réel » ${ }^{29}$ ?

$24 \quad$ Pline l'Ancien, $H N$, XII, 100.

25 Aristote, Histoire des animaux, I, 13.

26 Martial, Ép., XIV, 27.

27 Hinds S., art. cit., p. 116-118.

$28 C f$. la lecture $\dot{\alpha}[\pi] \alpha \lambda(\alpha i)$ proposée par W. Luppe pour les Scholia Londinensia 11 à Callimaque, Aitia, I, fr. 1 Pf, v. 11.

Cf. Martial, Ép., IX, 37, 4-5 qui parodie l'image élégiaque du personnage allongé dans son lit pour représenter la vieille Galla qui, après s'être fardée et parée de faux cheveux, dort « remisée dans cent pyxides et (dont le) visage fait chambre à part » (et iaceas centum condita pyxidibus, / nec tecum facies tua dormiat,). La résonance ovidienne mérite, je pense, d'être notée : cf. Ovide, Rem., 481 (nec mecum dormiat ulla) et, peutêtre, même si la valeur du subjonctif iaceas n'est pas la même chez Martial, Ovide, L'Art d'aimer, III, 265 (inque tuo iaceas quantulacumque toro). Que Martial s'amuse ici à détourner le modèle ovidien est à mon sens confirmé par la réponse, quelques vers plus loin (IX, 37, 7) à Ovide, Fastes, V, 57 (Magna fuit quondam capitis reuerentia cani). Ce dernier clin-d'œil à Ovide est signalé par Siedschlag E., art. cit., p. 156. 
Si Martial se joue en plusieurs points de ses Apophoreta des élégies didactiques d'Ovide, faut-il penser qu'il répondait aussi, en d'autres épigrammes, au modèle détourné par Ovide dans ses Medicamina, à savoir les Géorgiques de Virgile ${ }^{30}$ ? Il paraît difficile d'en juger, même si T. J. Leary a suggéré que les épigrammes des Xenia qui traitent des vins s'inspiraient en partie du catalogue des vins qui figure dans le livre 2 des Géorgiques ${ }^{31}$. L'hypotexte virgilien ou pseudovirgilien est présent en plusieurs points et généralement associé à l'univers bucolique : l'une des allusions les plus nettes figure dans le poème sur la chèvre en XIII, 98 ${ }^{32}$. Martial associe aussi avec humour les fromages de Trebula au souvenir de Virgile : Trebula nos genuit est en effet un calque du célèbre Mantua me genuit de l'épitaphe de Virgile ${ }^{33}$. L'univers bucolique est ainsi clairement rattaché à la figure de Virgile. Cette présentation du couple Virgile-Ovide semble suggérer que le premier se rattache à une forme de frugalité et à l'univers bucolique quand le second correspondrait à des images de raffinement cosmétique complexe.

Enfin, remarquons qu'une référence à l'univers des Bucoliques de Virgile est peut-être présente dans la toute dernière épigramme des Apophoreta : le surgite adressé aux lecteurs pour leur signifier que leurs occupations habituelles vont reprendre et que les Saturnales sont finies peut évoquer, entre autres modèles, le surgamus du vers 75 de la dixième eclogue, qui correspond à la venue du soir et à l'arrêt du chant ${ }^{34}$. On peut cependant citer d'autres modèles, comme les fins successives des trois livres de l'Orator de Cicéron où les protagonistes se lèvent et interrompent ainsi leur dialogue (I, 265 ; II, 367 ; III, 230). Cet autre modèle est peut-être d'autant plus important pour l'épigramme de conclusion des Apophoreta que l'adipata, beignet réputé très gras, semble devoir introduire, comme l'a souligné A. Barchiesi, une réflexion méta-littéraire, puisque cette pâtisserie avait été utilisée par Cicéron, comme métaphore, pour évoquer les dérives d'un asianisme huileux ${ }^{35}$.

Pour compléter ces quelques remarques sur la place du modèle virgilien dans le projet littéraire des Xenia et des Apophoreta, il faut enfin souligner que le modèle virgilien est notamment convoqué dans des épigrammes présentant un lien avec l'empereur ou la politique impériale $^{36}$. Ce choix s'explique naturellement par la volonté de privilégier le couple VirgileOctave comme modèle pour les rapports entre le poète et l'empereur et de célébrer Domitien comme restaurateur de la politique augustéenne. Ainsi dans l'épigramme 124, qui renvoie à la mesure que Domitien avait prise pour imposer le port de la toge pendant les spectacles :

Sur ce point, voir surtout Watson P. A., 2001, art. cit.

Martial, Ép., XIII, 106-125 et Virgile, Géorgiques II, 89-102. Pour le rapport possible entre ces deux passages, voir Leary T. J., Martial Book XIV : The Apophoreta. Text with introduction and commentary, Londres, Duckworth, 1996, p. 15. Contra, Grewing F., compte rendu de T. J. Leary, Martial Book XIII : The Xenia. Text with introduction and commentary, Londres, Duckworth, 2001, Bryn Mawr Classical Review, 2002.08.38 [http://bmcr.brynmawr.edu/2002/2002-08-38.html].

Cf. Virgile, Bucoliques, I, 76.

Cf. Donat. vita Verg. 36. Pour cette suggestion, voir Leary T. J., op. cit., 2001, p. 83 ; Grewing F., art. cit. ; Holzberg N., Martial und das antike Epigramm, Darmstadt, 2002, p. 45-46.

Sur les modèles possibles du « surgite » de l'épigramme XIV, 123, voir Citroni M., art. cit., p. 211.

Barchiesi A., "The Search for the Perfect Book : A PS to the New Posidippus », dans K. Gutzwiller (dir.), The New Posidippus. A Hellenistic Poetry Book, Oxford, Oxford University Press, 2005, p. 320-342, spéc. p. 328-329.

Voir infra le commentaire des épigrammes relatives aux statuettes représentant Hercule (XIV, 177-178). 
CXXIV. Toga

Romanos rerum dominos gentemque togatam

Ille facit, magno qui dedit astra patri.

\section{Une toge.}

Celui qui offrit les astres au grand empereur que fut son père rend les Romains maîtres du monde et leur fait porter la toge.

Le vers Romanos rerum dominos gentemque togatam (« les Romains maîtres du monde et peuple portant la toge ») est une citation de Virgile, qui attribue ces paroles à Jupiter annonçant que Junon et lui protégeront le peuple romain ${ }^{37}$. L'épigramme permet donc de comparer implicitement Domitien au père des dieux. Mais elle recèle aussi une allusion historique, puisque c'est en citant ce même vers qu'Auguste avait rendu le port de la toge obligatoire dans toute la zone du forum ${ }^{38}$. Une simple réminiscence littéraire permettait ainsi de comparer implicitement Domitien à Auguste et à Jupiter.

On comprend aisément que Martial privilégie le modèle virgilien pour évoquer la personne de l'empereur, mais ce choix est peut-être d'autant plus naturel pour l'épigrammatiste que le Virgile auquel il se réfère n'est pas seulement l'auteur de l'Énéide, des Géorgiques et des Bucoliques, mais aussi, et surtout, l'auteur du Culex, poème où il reconnaît une forme de lusus juvénile $^{39}$ et qui devait être d'autant plus intéressant pour lui qu'il s'agit d'un lusus explicitement adressé à l'empereur, comme en témoigne sa préface ${ }^{40}$. "Virgile » fournissait ainsi, aux yeux de Martial, l'exemple d'une poésie légère adressée au pouvoir et acceptée par lui.

\section{Des livres comme apophoreta}

Pour étudier le canon littéraire auquel se réfère Martial, il est particulièrement intéressant de se pencher sur les pièces 183 à 196 des Apophoreta qui correspondent à la description lapidaire de livres (volumina et codices) offerts à l'occasion des Saturnales ${ }^{41}$. Ces épigrammes

37 Virgile, Énéide, I, 282. Pour la citation de ce vers chez Martial, voir par exemple Spaeth Jr., J. W., art. cit., p. 26.

Suétone, Vie d'Auguste, 40, 8.

Martial, Ép. XIV, 185 : Vergili Culex. / Accipe facundi Culicem, studiose, Maronis, / Ne nucibus positis ARMA VIRVMQUE legas. ("Le Moucheron de Virgile. Reçois, ô diligent lecteur, le Moucheron de l'éloquent Virgile : ceci, pour ne pas lire « les armes et l'homme » alors que tu as à peine délaissé les noix/badinages. »). Sur la traduction de facundus, voir toutefois les remarques d'A. Deremetz, ici-même.

Culex, 1-10.

Sur ces poèmes, voir notamment Roman L., « The Representation of Literary Materiality in Martial's Epigrams », Journal of Roman Studies, n 91, 2001, p. 113-145 ; Prioux É., Petits musées en vers. Épigramme et discours sur les collections antiques, Paris, CTHS/INHA, 2008, p. 311-328. 
évoquent dans l'ordre : la Batrachomyomachie épopée miniature et parodique qui passait pour être l'œuvre d'Homère (183), un codex contenant l'Iliade et l'Odyssée (184), le Culex que Martial attribue à Virgile (185), l'Énéide (186), la Thaïs de Ménandre (187), un Cicéron (188), la Monobyblos de Properce (189), l'Ab Urbe condita de Tite-Live (190), un Salluste (191), les Métamorphoses d'Ovide (192), les Élégies de Tibulle (193), un Lucain (194), un Catulle (les Carmina?) (195) et le traité Du bon usage des eaux froides de Calvus (196).

Comme dans le reste du volume, il faut supposer une alternance binaire entre la description d'un présent visant un riche destinataire et celle d'un présent destiné à un pauvre. Le cycle des descriptions de livre est enserré entre un lot du pauvre (182 : figurine de bossu en argile) et un lot du riche (197: des mules naines), ce qui signifie qu'il est a priori fondé sur un va et vient régulier entre lots du riche et du pauvre. Dans la mesure où 182 est un lot du pauvre, 183 doit être un lot du riche. Martial aurait donc malicieusement associé les grands poèmes épiques et les œuvres de grande ampleur (Iliade et Odyssée, Énéide) aux " lots du pauvre », tandis que les pièces parodiques et humoristiques (Batrachomyomachie, Culex) et les œuvres les plus courtes sont considérées comme des lots du riche. Dans les quatre premières pièces, Martial entend clairement établir un parallélisme strict entre Homère et Virgile, un parallélisme qui se réfère certainement à l'idée selon laquelle les deux auteurs ont excellé dans le style moyen par leur habileté à embrasser tous les genres. Les épigrammes 183 à 192 respectent en outre une alternance entre volumes de papyrus et manuscrits sur parchemin, mais cette alternance ne se maintient pas jusqu'à la fin du cycle puisque le support du manuscrit de Lucain (194) est inconnu et que le traité de Calvus (196) est inscrit sur papyrus. Le classement par support n'est donc pas systématique et l'on remarque aussi que les œuvres grecques sont mêlées aux œuvres latines. On peut aussi relever un classement par genre mais qui n'est pas systématique : les deux historiens sont évoqués l'un après l'autre, les épopées sont rassemblées dans les pièces paires (lots du pauvres).

Face à cette tension entre classement et désordre, entre unité du cycle et dispersion, il me semble que l'une des voies les plus probantes pour rendre compte de l'ordre des épigrammes 183 à 196 consiste à lire ce cycle comme un exposé humoristique des positions esthétiques et des préférences littéraires de Martial.

\begin{tabular}{|c|c|}
\hline sortes diuitis & sortes pauperis \\
\hline 183 Homeri Batrachomachia & 184 Homerus in pugillaribus membraneis \\
\hline 185 Vergili Culex & 186 Vergilius in membranis \\
\hline 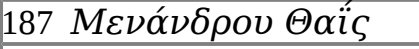 & 188 Cicero in membranis \\
\hline 189 Monobyblos Properti & 190 Titus Liuius in membranis \\
\hline 191 Sallustius & 192 Ouidi Metamorphosis in membranis \\
\hline 193 Tibullus & 194 Lucanus \\
\hline 195 Catullus & 196 Calui de aquae frigidae usu \\
\hline
\end{tabular}

Tableau 1: Alternance supposée entre les «lots du riche» et les «lots du pauvre» dans les épigrammes 183-196. 
Comme nous l'avons vu, les épigrammes paires renvoient à des œuvres en plusieurs livres ou à des auteurs particulièrement prolixes. Martial joue en particulier sur l'opposition entre la petitesse du support des feuillets de parchemin (pugillares membranei) et l'immensité des œuvres inscrites sur ces petits feuillets de parchemin: «dans de multiples feuillets de parchemin » (184), « l'immense Virgile » (186), " considère que tu vas parcourir un long chemin avec Cicéron » (188), « l'énorme Tite-Live, que ma bibliothèque ne suffit pas à contenir tout entier » (190), " cette masse, composée d'une multiplicité de pages, recèle les quinze chants d'Ovide » (192). Si les épigrammes impaires sont toutes conçues sous forme élogieuse, les épigrammes paires sont marquées par une évolution : les épigrammes 184, 186, 188, 190 et 192 évoquent, sous des formes variées, l'ampleur des œuvres que les livres sont susceptibles de renfermer. L'épigramme 194 évoque le jugement peu élogieux que certains critiques formulent sur l'œuvre de Lucain et l'épigramme 196, dernière pièce du cycle, tourne en dérision l'œuvre de Calvus :

CXCVI. Calui de aquae frigidae usu.

Haec tibi quae fontes et aquarum nomina dicit, Ipsa suas melius charta natabat aquas.

196. Calvus, Le Bon usage de l'eau froide.

Ces feuillets qui t'enseignent le nom des sources et des rivières auraient mieux fait de continuer à baigner dans les eaux qu'ils décrivent.

Dans cette épigramme, une œuvre de Calvus est muée en apophoreton exécrable, sur le modèle de l'anthologie offerte par Calvus à Catulle dans le Carmen 14 de ce dernier ${ }^{42}$.

L'opposition entre les épigrammes impaires et les épigrammes paires est soulignée par les jeux de réponse qui lient les épigrammes de l'une et l'autre série. On comparera notamment l'épigramme sur Tite-Live au poème qui célèbre les amours de Tibulle :

CXC. Titus Liuius in membranis

Pellibus exiguis artatur Liuius ingens, Quem mea non totum bibliotheca capit.

190. Tite-Live sur parchemin

L'énorme Live se trouve comprimé dans de minces feuilles de parchemin, lui que ma bibliothèque ne suffit pas à contenir tout entier.

$42 \quad$ Martial tourne cependant volontiers en dérision ses propres recueils, apophoreton dont la lecture peut-être abrégée à loisir et qui pourra être mué en cornet pour les olives ou les fritures à emporter : Ép. XIII, 1, $1-3$; 2 , 8 ; 3, 3-4 et 7-8 ; XIV, 1, 7-8 ; 2, 3-4. 
CXCIII. Tibullus

Ussit amatorem Nemesis lasciua Tibullum, In tota iuuit quem nihil esse domo.

193. Tibulle

La voluptueuse Némésis a fait brûler Tibulle, son amant, qui fut heureux de n'être plus rien dans toute sa maison ${ }^{43}$.

L'envahissant Tite-Live s'oppose à Tibulle, qui souhaite être anéanti, afin de laisser toute licence et tout pouvoir à sa maîtresse. Le motif du poète réduit à néant nous renvoie

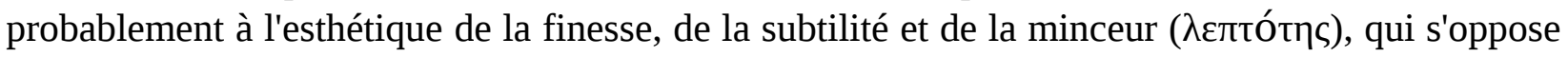

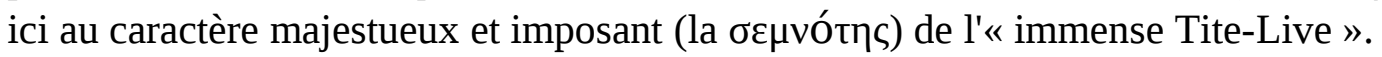

La cohérence du cycle vient de l'emploi d'un réseau de métaphores qui permettent de décoder le discours esthétique de Martial. La métaphore des longues routes (longas uias) parcourues avec Cicéron (XIV, 186) rappelle une célèbre image métapoétique et l'on peut songer qu'elle s'oppose à des chemins plus étroits et plus courts ${ }^{44}$. Les pièces impaires présentent volontiers des figures d'enfants ou de jeunes poètes qui rappellent des images callimachéennes ${ }^{45}$. Le cycle se clôt enfin sur une réflexion peu amène à l'endroit du traité $D u$ bon usage des eaux froides de Calvus. L'image de l'eau froide et du papyrus du Nil est en elle-même intéressante : elle conduit à souligner l'alexandrinisme de Calvus et ses liens avec l'œuvre de Callimaque, pris à la fois comme auteur de traités sur les fleuves ${ }^{46}$ et comme poète comparant son œuvre à l'eau fraîche d'une source ${ }^{47}$. Le motif de l'eau froide rappelle sous une forme banalisée le motif de la source fraîche où s'abreuve le poète ou encore celui de la controverse entre buveurs d'eau (hydropotai) et buveurs de vin (oinopotai) ${ }^{48}$; l'ironie est peut-être d'autant plus forte que Martial

43 Le pentamètre de l'épigramme 193 s'inspire de Tibulle, Élégies, I, 5, 29-30: Illa regat cunctos, illi sint omnia curae : / at iuuet in tota me nihil esse domo («Qu'elle commande à tous, qu'elle prenne soin de tout : je serai heureux de n'être plus rien dans toute ma maison »).

44

L'image du chemin est notamment employée par Pindare (voir par exemple Pythiques, IV, 247). Elle sera reprise à l'époque hellénistique : le locus classicus est Callimaque, Aitia, fr. 1 Pf, v. 25-26. Pour des remarques sur ce type d'imagerie, voir Worman N., « Bodies and Topographies in Ancient Stylistic Theory », dans Th. Fögen - M. M. Lee (dir.), Bodies and Boundaries in Graeco-Roman Antiquity, Berlin - New York, Walter de Gruyter, 2009, p. 45-62.

Sur cette image, voir par exemple Ambühl A., Kinder und junge Helden. Innovative Aspekte des Umgangs mit der literarischen Tradition bei Kallimachos, Louvain, Peeters, 2005.

Voir Prioux É., " On the Oddities and Wonders of Italy : When Hellenistic Poets Look Westward », dans M. A. Harder, R. Regtuit, G. C. Wakker (dir.), Nature and Science in Hellenistic Poetry, Louvain, Peeters, 2009, p. 121-148, spéc. n. 6, avec la bibliographie antérieure.

Voir par exemple Poliakoff M., "Nectar, Springs, and the Sea: Critical Terminology in Pindar and Callimachus », ZPE, n 39, 1980, p. 41-47.

L'association entre discours stylistique et ivresse est notamment attestée au sujet de Cratinos, puis reprise par la tradition postérieure, à l'époque hellénistique puis impériale, sous la forme d'une opposition entre buveurs d'eau et buveurs de vin (dans le fr. 544 Pf, Callimaque qualifie ainsi Cratinos de $\mu \varepsilon \theta 0 \pi \lambda \eta_{\eta} \xi$ ). Voir par exemple Worman N., art. cit., p. 53-54. 
pouvait difficilement ignorer que l'un des usages les plus connus de l'eau froide était d'être un anti-aphrodisiaque puissant ${ }^{49}$, ce qui oppose cette dernière épigramme à la figure des poètes élégiaques consumés par l'amour. Le cycle de Martial suggère donc une antithèse entre deux héritages alexandrins : du côté des lots du riche, on trouve Properce, le Callimaque romain, mais aussi un goût pour l'image du jeune poète ou du poète comparé à un enfant, suivant un motif qui rappelle par exemple la préface des Aitia ${ }^{50}$; on trouve aussi Catulle dont Martial imitera tant la verve sans pourtant s'attacher à ce qui définit l'esthétique néotérique (le goût pour les thématiques sentimentales, pour le limae labor et pour la posture d'un poète «menu »). Du côté des lots du pauvre, on rencontre les Métamorphoses d'Ovide, dont les liens avec les Aitia de Callimaque seront ultérieurement suggérés par Martial ${ }^{51}$, mais aussi un Calvus callimachéen, représentant majeur de l'atticisme dont il est aussi l'un des principaux théoriciens et dont le traité technique sur les eaux froides est rejeté avec dédain. Ce rejet correspond sans doute, de la part de Martial, à un refus des afféteries efféminées des néotériques qui, aux yeux de Martial, provoqueraient des applaudissements faciles mais n'auraient aucune prise sur la réalité52.

Mais la principale image dont Martial se sert pour formuler ses positions esthétiques tient apparemment à la matérialité des livres, à leur multiplicité ou à leur unicité, à leur épaisseur, à leur forme de codex ou de volumen, à leur facture en papyrus ou en parchemin. L. Roman a ainsi suggéré que l'adjectif multiplex qui sert à décrire la structure des codices formés de multiples feuillets $(184,192)$ possédait en réalité une signification métapoétique : l'adjectif est utilisé au sujet des épopées d'Homère et des Métamorphoses d'Ovide. Dans le cas d'Homère l'adjectif pourrait évoquer la structure narrative complexe de l'Odyssée avec ses analepses temporelles. Dans le cas des Métamorphoses, cet adjectif peut évoquer l'enchaînement complexe des récits mythologiques avec l'intervention de différents narrateurs intradiégétiques et de fréquents récits enchâssés. Parler de la matérialité du livre devient ainsi, pour Martial, un moyen détourné de commenter sa facture poétique ${ }^{53}$.

L'exposé de Martial présente plusieurs points de contact avec les conseils de lecture donnés par Quintilien dans l'Institution oratoire au chapitre X, 1. Les deux textes s'inscrivent, chacun à leur manière, dans la tradition hellénistique des « canons » (listes brèves d'auteurs ayant excellé dans un genre littéraire donné) ${ }^{54}$. Lorsque Martial retient Tite-Live et Salluste comme principaux historiens mais accorde la préférence à Salluste, il propose un jugement très proche de

49 Pour cette remarque, voir Mattiacci S., « Marziale e il neoterismo », dans A. Bonadeo - E. Romano (dir.), Dialogando con il passato. Permanenze e innovazioni nella cultura latina di età flavia, Florence, 2007, p. 177206, spéc. p. 183-184 et n. 26.

Callimaque, Aitia, fr. 1 Pf, v. 5.

Pour des critiques de la poésie mythologique, voir Martial, Ép., IV, 49 et X, 4. L'épigramme X, 4 oppose la poésie de Martial qui « a une odeur d'humanité » (hominem pagina nostra sapit) à la poésie mythologique sans lien avec la vie réelle et qui narre par exemple les histoires d'EEdipe et de Thyeste, de Médée et de Scylla et qu'il associe aux Aitia de Callimaque. Si la source des mythes que Martial cite ne semble pas correspondre à une seule œuvre mythologique mais à plusieurs d'entre elles, on peut souligner qu'il prend soin de parodier les techniques d'association de mythes que l'on peut rencontrer dans les Métamorphoses d'Ovide et que plusieurs des figures qu'il cite sont étroitement associées au poème ovidien. Voir Hinds S., art. cit., spéc. p. 136-139 et infra, p. ???.

52 Mattiacci S., art. cit.

53 Roman L., 2001, art. cit. 
celui de Quintilien ${ }^{55}$. Il en va de même pour le jugement sur Lucain dont Quintilien affirmera qu'il est un meilleur modèle pour les orateurs que pour les poètes ${ }^{56}$. Or, l'admiration sans réserve que Martial a pour Virgile et l'attitude mitigée qu'il a à l'endroit d'Ovide rejoignent elles aussi les positions exprimées par Quintilien qui, de Virgile, louait l'ensemble du corpus, mais, dans le cas d'Ovide, réservait ses éloges à la Médée qui ne nous est pas parvenue ${ }^{57}$.

La présentation qui est ici donnée des œuvres de Virgile et d'Ovide est donc à la fois l'expression de jugements partagés par une partie des contemporains de Martial, mais aussi

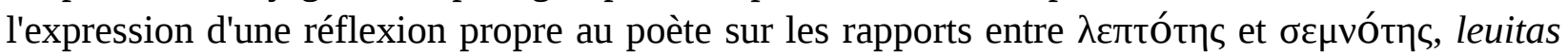
et grauitas, genus grande et genus tenue. La réflexion métapoétique semble en outre rejoindre une réflexion d'ordre rhétorique - idée qui ne nous est pas seulement suggérée par les liens avec le programme d'études défendu par Quintilien, mais aussi par le fait que Martial regarde vers des figures comme Calvus, qui fut à la fois une figure de proue de la poésie néotérique poète et un représentant éminent de l'atticisme dont il fut sans doute l'un des principaux théoriciens. L'intérêt de Martial pour la controverse entre atticisme et asianisme est par ailleurs confirmé par le fait qu'il évoque, quelques épigrammes plus haut, les choix esthétiques de Brutus ${ }^{58}$ et qu'il clôt le recueil des Apophoreta avec une référence à l'adipata, image, pour Cicéron, d'un asianisme huileux ${ }^{59}$. On devine donc, dans ces recueils en apparence assez anodins, une réflexion ambitieuse sur la littérature.

\section{Quand Virgile et Ovide sont directement confrontés}

Après ces quelques remarques sur la présence d'une réflexion métapoétique ambitieuse distillée par petites touches et souvent sous forme de métaphores dans les épigrammes de Martial, j'aimerais revenir sur les quelques passages où Virgile et Ovide sont directement confrontés ou rapprochés dans les Xenia et les Apophoreta, à savoir l'épigramme XIII, 96 sur le cerf domestiqué offert en guise de gibier dans les Xenia et le cycle d'ecphraseis de peintures, statuettes et reliefs rassemblé dans les Apophoreta (XIV, 170-182).

En XIII, 96, Martial rapproche deux passages - l'épisode du cerf de Silvia tiré de l'Énéide (VII, 475-539) et celui du cerf de Cyparissos tiré des Métamorphoses (X, 106-142) -, qui présentent entre eux un rapport d'intertextualité ${ }^{60}$ : C. Connors a notamment montré comme le passage ovidien exagère tous les traits de l'épisode virgilien - au lieu d'une Silvia tressant des

54

Sur les jugements de Quintilien et leurs liens avec la tradition des « canons », voir Citroni M., « Finalità e struttura della rassegna degli scrittori greci e latini in Quintiliano », dans F. Gasti - G. Mazzoli (dir.), Modelli letterari e ideologia nell'età flavia. Atti della III Giornata ghisleriana di Filologia classica (Pavia, 30-31 ottobre 2003), Côme - Pavie, 2005, p. 15-38.

Quintilien, Institution oratoire, II, 5, 19.

Quintilien, IO, X, 1, 10 .

Quintilien loue sans réserve sont Virgile (IO, X, 1, 85-86) et Tibulle (93), suivis par des figures placées très légèrement en retrait : Properce $(93)$, Horace $(94,96)$, et enfin Varius et Ovide, dont les corpus sont soumis à une sélection et qui sont respectivement loués pour le Thyeste du premier et pour la Médée du second (98).

Martial, Ép., XIV, 171.

Voir supra p. ???.

Sur ce distique, voir, ici-même, la conclusion de l'article d'A. Deremetz. 
couronnes de fleurs pour son cerf, on voit ainsi Cyparissos attacher des bijoux et des gemmes aux bois de son animal ${ }^{61}$. Ovide aurait mis en évidence la manière dont l'épisode virgilien touchait aux limites du genre épique par sa leuitas et son décalage avec les combats qui suivent la mort du cerf. En transformant les fleurs en gemmes, Ovide force et exagère nettement certains traits du récit virgilien qui pouvaient passer pour être en porte à faux par rapport au reste du récit épique. Il aurait ainsi souligné comme le cerf de Silvia se situait, en quelque sorte, aux marges du genre épique. Connors souligne aussi le fait que, chez Ovide, Apollon intervient pour exhorter Cyparissos à leuiter pro materia (dolere), autrement dit manifester une douleur proportionnée à la perte d'un animal familier (par opposition à la famille de Silvia qui vengera par les armes la mort de l'animal). Or, ce conseil donné par Apollon peut évoquer, d'un point de vue métapoétique, une recusatio similaire à celles qui figurent en plusieurs point des Bucoliques de Virgile $^{62}$. Si cette lecture proposée par Connors est juste, Ovide pointerait donc la nature paradoxale de l'épisode de Silvia, qui introduit une forme de leuitas dans le poème épique, mais il soulignerait aussi l'originalité de son propre choix qui consiste à inclure, comme en tant d'autres lieux des Métamorphoses, un véritable épyllion au sein de l'épopée. Martial, dont le propos initial était de comparer le sort d'un cerf domestiqué qu'on destine à la consommation aux précédents mythologiques évoqués par Virgile et Ovide, a-t-il été sensible à ce jeu complexe des deux poètes et fait-il ici allusion à des questions esthétiques qui l'intéressaient de près ? Il est difficile d'en juger, mais il me semble intéressant de remarquer que les passages épiques qui retiennent ici l'attention de l'épigrammatiste sont ceux qui présentent des rapports très complexes avec la tradition alexandrine, ceux où les frontières génériques sont peut-être les plus labiles et où certaines formes d'épyllia ou, dans le cas de Virgile, un certain univers bucolique font le plus nettement irruption dans l'épopée. Ce constat rejoint une remarque formulée par S. Hinds au sujet d'autres effets d'intertextualité : « (...) Martial has managed to evoke something Martialian in his epic model - (...) a moment when epic pretension and pomposity come in for the kind of deflation which at times (...) can seem to be the epigrammatist's programmatic precondition for any allusion to the epic genre. ${ }^{63}$

Une confrontation à la fois assez nette et significative entre le modèle ovidien et le modèle virgilien intervient aussi dans le cycle d'ecphraseis que Martial rassemble dans les pièces 170 à 182 des Apophoreta. Il s'agit de descriptions d'œuvres d'art (statuettes, tableaux, reliefs) qui présentent bien souvent un lien thématique avec l'idéologie impériale et qui semblent évoquer, dans certains cas, des œuvres d'art rassemblées dans le Templum nouum Divi Augusti qui fut peut-être restauré sous les Flaviens ${ }^{64}$. Comme je l'ai montré dans un travail antérieur, l'un des enjeux de ce court cycle semble en tous cas résider dans la volonté de comparer les figures

61 Connors C., art. cit., p. 8-10.

62 Connors C., ibid., p. 11-12.

63 Hinds S., art. cit., p. 145.

64 Cette hypothèse fut d'abord proposée par K. Lehmann, « A Roman Poet Visits a Museum », Hesperia. Journal of the American School of Classical Studies at Athens, $\mathrm{n}^{\circ}$ 14, 1945, p. 259-269, à partir de la convergence entre les thèmes mythologiques cités par Martial et les quelques notices concernant les collections de ce temple qui fut vraisemblablement restauré sous les Flaviens. S'il est impossible d'infirmer ou de confirmer cette hypothèse, il n'en reste pas moins que les thèmes iconographiques sélectionnés par Martial pourraient tout à fait correspondre à ceux d'une collection artistique destinée à célébrer Domitien comme restaurateur et véritable héritier de la politique augustéenne : voir Prioux É., op. cit., p. 267-273. 
d'Auguste et Domitien, pour mettre en évidence le fait que les Flaviens sont les véritables successeurs d'Auguste, les restaurateurs de sa politique, mais que Domitien l'emporte aussi sur son modèle ${ }^{65}$. Il me paraît assez significatif que ce cycle d'épigrammes contienne notamment deux diptyques centrés l'un sur la figure d'Apollon (XIV, 172-173), l'autre sur la figure d'Hercule (XIV, 177-178), autrement dit sur les deux divinités qui avaient pu servir de modèle aux empereurs romains : Apollon pouvait rappeler la figure d'Auguste, mais aussi, sous un jour plus sombre, les comparaisons qu'un Caligula ou un Néron avaient volontiers mises en place entre ce dieu et leur propre personne, tandis que Domitien était connu pour se faire représenter sous les traits d'Hercule. Les deux diptyques sont reliés par un système de rappels thématiques et stylistiques. Le Sauroctone (172) et l'Hyacinthe (173) forment ainsi un diptyque centré sur la figure d'Apollon : alors que le jeune Apollon tue volontairement le lézard qui s'approche de lui, le dieu adulte tue involontairement le jeune Hyacinthe dont les yeux se détournent du disque. Martial s'est plu à opposer les deux images, en soulignant l'antithèse entre le geste d'Hyacinthe, qui « détourne ses yeux mourants du disque haï » (Flectit ab inuiso morientia lumina disco: XIV $, 173,1$ ), et le mouvement du lézard, qui « glisse vers » son bourreau (Ad te reptanti : XIV, $172,1)$.

De l'autre côté, Martial se plaît à souligner une analogie entre deux figures d'Hercule : l'Hercule en terre cuite (XIV, 178) et l'Hercule enfant terrassant les serpents (XIV, 177), deux œuvres qui renvoient au motif de la fragilité trompeuse. Le sujet de l'Hercule en bronze corinthien (XIV, 177) est un petit enfant et la matière dans laquelle est sculpté l'Hercule en argile est synonyme de fragilité, mais les deux images se montrent dignes du héros qu'elles représentent (XIV, 177, 2 et 178, 2). Or, ces deux images sont aussi reliées entre elles par leur commune référence à l'Énéide. L'épigramme sur l'Hercule en bronze corinthien se souvient en effet de trois hypotextes : l'Idylle XXIV de Théocrite sur l'enfance d'Héraclès, un poème très probablement destiné à célébrer Ptolémée II, puisque les «teneras... manus » du petit Hercule étranglant les

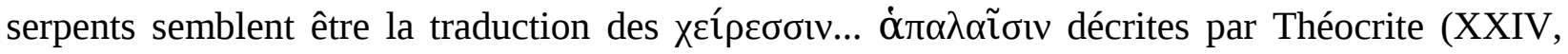
$55)^{66}$, l'hymne à Hercule du chant VIII de l'Énéide, puisque le vers Elidit geminos infans nec respicit anguis se souvient du vers monstra manu geminosque premens eliserit angues (Én. VIII, 289) ${ }^{67}$, et enfin de la victoire d'Auguste sur Cléopâtre et Antoine représentée sur le bouclier d'Énée, toujours dans le chant VIII de l'Énéide (necdum etiam geminos a tergo respicit anguis) ${ }^{68}$. L'Hercule en terre cuite se souvient, quant à lui, d'un autre passage du chant VIII de l'Énéide : celui où Évandre engage Énée à accepter son hospitalité puisque son humble demeure fut un séjour qui agréa, jadis, à Hercule et que condescendre à accepter l'invitation d'un pauvre homme fera donc d'Énée l'égal d'Hercule ${ }^{69}$. Encore une fois, le passage que Martial sélectionne dans le poème épique se distingue par ses rapports complexes avec la poésie alexandrine et avec une forme d'altération des attentes génériques: Évandre nous parle d'un Hercule humble,

\footnotetext{
65 Prioux É., ibid., p. 253-335.

66 Prioux É., ibid., p. 292-295.

67 Spaeth Jr., J. W., art. cit., p. 27.

68 Spaeth Jr., J. W., ibid., p. 27.

69 Virgile, Énéide, VIII, 359-368 avec en particulier les vers 864-865 : et te quoque dignum / finge deo (« toi aussi rends-toi digne du statut divin en te conformant à ce modèle »). Sur l'effet d'intertextualité qui lie l'épigramme sur l'Hercules fictilis à ce passage de l'Énéide, voir S. Hinds, art. cit., p. 145-146.
} 
s'accommodant d'un pauvre séjour et le modèle évident de ce passage devait être l'élégie de Callimaque sur la victoire de Bérénice, élégie adressée à une reine lagide mais qui décrit Hercule reçu par Molorchos dans sa masure ceinte de ronces et menacée par l'avidité des souris ${ }^{70}$. Hercule, le dieu préféré de Domitien, est un donc un dieu qui, tout en conservant sa force et sa dignité hérö̈que, accepte d'être reçu dans de pauvres demeures et d'être représenté dans des images qui font de lui un enfant aux tendres mains ou qui le mue en figurine de terre cuite. L'implication que l'on peut deviner est que ce dieu, et l'empereur qui s'identifie à lui, pourraient agréer le don des épigrammes de Martial, des poèmes de petite ampleur et de peu de prix. Le corollaire de ce premier constat est que, pour célébrer le dieu Hercule (et, partant, l'empereur Domitien), Martial va intégrer dans l'épigramme des éléments de l'épopée virgilienne et transformer ainsi le genre poétique qu'il pratique. Le dieu de Domitien et l'épigrammatiste trouvent ainsi un terrain commun où la poétique de Martial et la célébration des actions héroïques de l'empereur ou de son dieu préféré peuvent se rejoindre, malgré l'humilité du genre pratiqué par Martial $^{71}$. Accepter un don humble fera d'Énée, mais peut-être aussi de Domitien, l'égal d'Hercule.

En face de ce diptyque consacré à Héraclès, on rencontre un autre diptyque consacré à Apollon, un dieu qui évoque bien sûr la figure d'Auguste. Or, ce nouveau diptyque se compose d'une part de l'épigramme sur le Sauroctone qui peut passer pour un pendant de l'Héraclès étranglant les serpents : dans les deux cas, la statue est en effet en bronze corinthien et elle représente un dieu juvénile tuant un reptile. Martial s'est d'ailleurs plu à souligner le parallèle entre les deux œuvres en mettant à chaque fois en valeur le détail de la main du jeune dieu ; digitis tuis («par tes doigts»: 172, 2), teneras... manus (« ses tendres mains »:177, 2). On peut toutefois remarquer la distance qui sépare ces deux figures puisque Héraclès se défend contre un monstre qui le menace, tandis que le Sauroctone tue, apparemment par jeu (puer insidiose), un lézard insignifiant. Si l'on se fie à cette présentation, la comparaison entre Héraclès enfant et Apollon enfant est donc clairement en faveur d'Héraclès et il était certainement de bon ton, après le règne de Néron, de présenter Hercule comme un modèle qui, pour le prince, était largement préférable à celui d'Apollon. Dans cette perspective, il me paraît intéressant de remarquer que l'autre image d'Apollon, le tableau avec Hyacinthe, est décrit en des termes empruntés aux Métamorphoses d'Ovide. On peut ainsi comparer l'expression Phoebi culpa dolorque («le remords et la douleur de Phébus » [Ép. XIV, 173]) à la plainte exprimée par Apollon dans le récit ovidien : tu dolor es facinusque meum (« tu es ma douleur et mon crime » [Métamorphoses, X, 198]) et quae mea culpa tamen (« et pourtant, quelle est ma faute ?» [Mét., X, 200]). Comme le remarque S. Hinds, Martial se plaît ici à concentrer en deux mots (culpa dolorque) plusieurs vers du récit ovidien, ce qui nous renvoie à l'esthétique de la synthesis chère à l'épigrammatiste ${ }^{72}$. Mais j'ajouterais que le fait d'associer la représentation de victimes d'Apollon (Hyacinthe et le lézard) à l'intertextualité ovidienne devait aussi évoquer indirectement, dans le contexte des allégories

\footnotetext{
70 SH 254-268 C.

71 De manière intéressante, Martial aura à nouveau recours à une réécriture de deux vers virgiliens relatifs à Hercule dans l'épigramme IX, 101 qui évoque la statue de Domitien-Hercule qui se trouvait dans le temple que l'empereur flavien avait dédié à sa divinité préférée sur la voie Appia : cf. Ép. IX, 101, v. 7-10 et Virgile, Én., VI, 802 et VII, 663. Voir Spaeth Jr., J. W., art. cit., p. 26-27.

72 Hinds S., art. cit., p. 141-144.
} 
politiques rassemblées dans ce cycle d'ecphraseis, l'image d'une victime célèbre d'Auguste: Ovide exilé pour un carmen qui était aussi un crimen. Si cette hypothèse de lecture est juste, Martial opposerait donc deux types de rapports entre le prince ou le dieu et les œuvres d'art (qu'il s'agisse de poèmes ou de représentations figurées). Cette opposition serait symbolisée par la comparaison qu'il établit, implicitement, entre deux dieux (Hercule et Apollon), mais aussi entre deux poètes (Virgile et Ovide).

\section{Virgile, Ovide et Martial dans les images métapoétiques des Xenia et des Apophoreta}

À ce point de mon exposé, je pense qu'il apparaît assez clairement que les commentaires que j'ai proposés plaident en faveur d'une présence assez affirmée de considérations d'ordre esthétique et métapoétique dans le garde-manger des Xenia et dans le bric à brac des Apophoreta. De fait, il me semble que nous devons prêter attention à l'intérêt récurrent que Martial prête à des objets qu'une longue tradition a étroitement associée au discours sur le style. Je trouve en effet très frappant qu'à côté d'objets isolés, comme le cure-oreille, le grattoir pour enlever les puces, le tablier, le saucisson ou l'éponge, qui visent à satisfaire des besoins corporels immédiats, quotidiens et parfois sordides et qui se rattachent tous à la poétique du réel ou au « parti-pris des choses » que les commentaires considèrent comme constitutifs de l'esthétique de Martial $^{73}$, se dessinent aussi des séries, organisées sous formes de petits cycles thématiques centrés sur des ustensiles qui sont, par ailleurs, employés de manière courante comme métaphores du style : les tablettes à écrire (XIV, 3-11; $19 ; 21)^{74}$, les jeux de hasard (XIV, 14-18) ${ }^{75}$, les oiseaux (XIV, 73-

73 Salemme C., Marziale e la «poetica » degli oggetti : struttura dell'epigramma di Marziale, Naples, Società editrice napoletana, 1976 et Marziale e la poesia delle cose, Naples, Loffredo, 2005 ; Laurens P., « La poétique de la langue ou la performance descriptive dans le livre VI de l'Anthologie grecque et dans les livres XIII et XIV de Martial », Revue de Philologie, n 66-2, 1992, p. 301-315.

Voir Roman L., 2006, art. cit., spéc. p. 381-388,qui identifie une section d'images métapoétiques courant dans les pièces XIV, 1 à 21.

Martial compare volontiers ses propres épigrammes écrites à l'occasion des Saturnales à des jeux de hasard, notamment à partir d'un jeux sur le mot nuces (badinages, mais aussi noix qui peuvent être utilisées pour jouer : XIII, 1, 5-8 et XIV, 1, 12). Sur le dé comme image métapoétique chez Martial, voir Roman L., 2006, art. cit., spéc. p. 381. L'image du dé avait déjà été employée par Méléagre pour caractériser, tout en la brocardant, la poétique d'Antipater de Sidon, que l'anthologiste présentait comme un buveur invétéré : Anthologie palatine VII, 428 = Méléagre de Gadara CXII G.-P. Voir supra p. ??? et n. ??. 
77, dont le moineau de Lesbie en XIV, 77), les coupes (XIV, 93-118) ${ }^{76}$, les étoffes (XIV, 124$143 ; 145)^{77}$ et les instruments de musique (XIV, 163-167) ${ }^{78}$.

Je n'entends pas dire que l'ensemble des Xenia ou des Apophoreta doivent être déchiffrés comme des allégories de la poésie : ce serait certainement une erreur grossière ! Mais il me semble que, ponctuellement, la convergence d'images métapoétiques et d'un vocabulaire couramment employé dans des contextes de critique littéraire doit nous inviter à prêter attention à la possibilité d'une interprétation métaphorique pour certains poèmes. Comme toute allégorie, ces poèmes possèdent un sens premier qui est parfaitement satisfaisant en l'état et il est tout à fait possible et même tentant de s'arrêter sur une lecture littérale du texte. Les pistes que je propose ici ne sont donc que des propositions de lecture, mais il me semble que certains poèmes nous livrent, à travers l'image d'objets, des représentations, par Martial, de sa propre poétique ou de celle de certains de ses prédécesseurs ou de ses contemporains ${ }^{79}$. Dans le passage sur les coupes, on trouve ainsi une invitation à ne pas dédaigner les humbles vases d'Arezzo en terre cuite (XIV, 98) qui préfigure les termes de l'épigramme sur l'humilité d'Hercule acceptant d'être représenté en terre cuite (XIV, 178). L'inventaire des présents du pauvre comprend encore des calices audaces (XIV, 94), coupes en verre de peu de prix (plebeia toreumata) qui ne craignent rien et qui sont peut-être une image appropriée de la poétique de Martial, des coupes faites en terre espagnole ${ }^{80}$ qui, elles non plus, ne craignent rien (XIV, 108), des coupes de Sorrente, qui, bien que faites de terre, ne sont pas uili de puluere natos, mais un leue toreuma (XIV, 102) ${ }^{81}$, ou encore la panaca, récipient utilisé à Vérone et qui permet d'introduire une allusion à Catulle ${ }^{82}$. À l'opposé, on y trouve également des coupes ornées de gemmes (XIV, 109), des ampoules qui pourraient aussi bien accueillir du vin que du parfum (XIV, 110), ou encore des œuvres de Mentor et de Mys ${ }^{83}$, deux toreuticiens dont les ciselures sont par ailleurs été citées dans des contextes métapoétiques

Sur les descriptions de coupes comme textes programmatiques dans la poésie hellénistique et romaine, voir Faber R., «Vergil Eclogue 3.37, Theocritus 1 and Hellenistic Ekphrasis », American Journal of Philology, ${ }^{\circ}$ 116, 1995, p. 411-417.

La comparaison entre texte et tissage est par exemple bien attestée dans la lyrique archaïque : voir Pindare, Néméennes, IV, 94 ; Olympiques, VI, 86-87 ; Bacchylide, V, 9-10. Pour l'application de lectures métapoétiques à des ecphraseis de vêtements, voir par exemple Faber R., "The Woven Garment as Literary Metaphor : The Peplos in Ciris 9-41 », dans J. Edmonson - A. Keith (dir.), Roman Dress and the Fabrics of Roman Culture, Toronto, University of Toronto Press, 2008, p. 205-216, spéc. 205-206 et 208-209.

Dans les Xenia, les cycles ne concernent pas seulement des produits usuellement considérés comme métapoétiques, mais il est intéressant de constater que le livre se clôt sur le miel (XIII, 104-105), le vin (XIII, 106-125), les parfums (XIII, 126) et des couronnes de roses (XIII, 127), qui sont à la fois un symbole approprié pour le banquet et pour l'ensemble d'un recueil poétique : le même procédé est employé avec une couronne civique en lierre adressée à Domitien pour clore le livre VIII (Ép., VIII, 82) : voir Henriksén Chr. (2004) : compte rendu de C. Schöffel, Martial, Buch 8. Einleitung, Text, Übersetzung, Kommentar, Stuttgart, Franz Steiner, 2002, Bryn Mawr Classical Review, 2004.01.21 : [http://bmcr.brynmawr.edu/2004/2004-01-21.html] ; Barchiesi A., art. cit., p. 325.

Pour la lecture d'une épigramme portant sur un fragment de la nef Argo (VII, 19) comme allégorie de la poétique de Valérius Flaccus, voir Zissos A., « Navigating Genres: Martial 7.19 and the Argonautica of Valerius Flaccus », The Classical Journal, n 99-4, 2004, p. 405-422.

Les coupes sont de Sagonte qui est, certes, bien éloignée de Bilbilis, mais je me demande si l'origine ibérique de ces coupes en terre ne suffit pas à établir un lien privilégié avec la figure de Martial.

Pour cette présentation qui assimile un vase réalisé dans un matériau non métallique à un produit d'orfèvrerie, on pourra comparer avec le modèle de l'Idylle I de Théocrite.

Voir Leary T. J., « Martial 14.100 : Panaca », The Classical Quarterly, n 47, 1997, p. 322-323. 
fameux, probablement pour évoquer la réduction des sujets épiques dans des œuvres miniatures ${ }^{84}$. La diversité même de ces coupes renvoie-t-elle à la diversité de l'œuvre de Martial capable d'embrasser dans ses épigrammes les présents des riches et des pauvres ${ }^{85}$ ?

De manière répétée, Martial insère dans ses trois premiers recueils des objets et des images qui évoquent des symboles métapoétiques chers à Ovide. Par trois fois, il mentionne, en termes ovidiens, des paons : l'un est est destiné à la consommation ${ }^{86}$, l'autre a vu sa queue a transformée en tapette à mouche ${ }^{87}$ (clin d'œil narquois à l'histoire d'Io gardée par Argus et poursuivie par un taon $?^{88}$ ), un autre encore sert de modèle pour l'ornement d'un lit ${ }^{89}$ ! Le paon, qui occupe bien sûr une place cardinale dans le récit des aventures d'Io des Métamorphoses, peut aussi rappeler le prologue des Medicamina faciei femineae où Ovide répond au soupçon d'immoralité planant sur l'art des cosmétiques en suggérant que le fait de se parer est, pour une femme, aussi naturel que l'est, chez le paon, celui de faire la roue devant les hommes, autrement dit devant des êtres pour lesquels il n'éprouve pas d'appétit sexuel ${ }^{90}$. Le paon est ainsi l'image même d'un art du maniement des cosmétiques qui veut être perçu non comme confinant au crimen, mais comme un prolongement de la nature ${ }^{91}$.

83

Martial, Ép., XIV, 93 (sur Mentor) et 95 (sur une coupe d'or ciselé) où l'art de Mys est associé à l'or de Galice, ce qui permet d'introduire une référence (autoréférentielle ?) à la péninsule ibérique chère à Martial.

Voir Properce, Élégies, III, 9, 13-14, avec, cependant, un contraste apparent entre les thèmes plus élevés des ciselures de Mentor et l'art plus ornemental de Mys. Nous manquons cependant d'éléments pour confirmer ou infirmer l'évaluation contrastée que Properce nous livre des deux artistes. Mys passe par ailleurs pour avoir utilisé comme modèles des dessins de Parrhasios, notamment pour sa représentation du combat entre les Centaures et les Lapithes (Pausanias, Description de la Grèce, I, 28, 2 ; Athénée, Deipnosophistes, IX, 728b) ; or, Parrhasios est associé de manière répétée avec les catégories stylistiques que sont la mollitia, le $\gamma \lambda \alpha \varphi$ pó $\nu$ et l'åßpótns (voir Athénée, Deipnosophistes, XII, 543c et Pline l'Ancien, HN, XXXV, 69 et Plutarque, De gloria Athenensium, 2. L'intérêt exprimé pour les toreuticiens dans les textes hellénistiques et romains tient sans doute en partie à leur capacité de réprésenter les sujets épiques sous une forme miniature : certains témoignages suggèrent en effet que l'art des orfèvres s'apparente à celui des auteurs d'épyllia. Voir Faber R., " The Literary Metaphor of the Chisel (Tornus) in Eclogue 3.38 », Hermes, n 128, 2000, p. 375-379 et AP IX, 545 (de Crinagoras) sur l'Hécalè de Callimaque comme торєuтòv ह̌noৎ.

Martial reviendra sur les figures de Mys et de Mentor pour décrire la coupe d'or qui lui aurait été offerte en cadeau par Instantius Rufus : de manière peut-être programmatique, cette coupe présente l'image d'un bouc dont la toison rivalise avec celle du bélier de Phrixos, ce qui place (comiquement ?) le bouc au-dessus de la toison d'or, symbole propre à évoquer le monde de l'épopée et notamment le cycle des Argonautes (le bélier de Phrixos est d'ailleurs l'un des motifs ornant le manteau de Jason dans la célèbre ecphrasis des Argonautiques d'Apollonios de Rhodes : I, 763-767).

Martial, Ép., XIV, 1, 5.

Martial, Ép., XIII, 70. Comparer v. 1 (gemmantis explicat alas) à Ovide, Medic., 33-34 (laudatas [...] pinnas / explicat), ainsi qu'à Mét., I, 723 (gemmis caudam [...] inplet) et, comme le remarque Zingerle A., op. cit., p. 33, à Am., II, 6, 65 (explicat ipsa suas ales Iunonia pinnas) - extrait de l'élégie sur la mort du perroquet de Corinne.

Martial, Ép., XIV, 67. Comparer v. 2 (superba) et Ovide, Medic., 34 (superbit).

La tapette en queue de paon est en outre suivie d'une tapette en queue de bœuf, ce qui peut rappeler l'association entre Argus et la vache Io : XIII, 68 (71). Dans cette autre épigramme, Siedschlag E., art. cit., décèle une réminiscence probable d'Ovide, $A m$., III, 2, 41-42.

Martial, Ép., XIV, 85. Comparer v. 1-2 (pictis pulcherrima pinnis / nunc Iunonis auis) et Ovide, Medic., 3334 (laudatas [...] uolucris Iunonia pinnas / [...] auis) ou Ovide, Am., III, 2, 41-42.

Voir Ovide, Medic., 31-34 et Heldmann K., art. cit. 
Les vêtements et étoffes sont eux aussi susceptibles de constituer des images métapoétiques ; or, on rencontre parmi elles la toge décrite en termes virgiliens (XIV, 124), et, à l'opposé, des étoffes chamarrées ou brodées comme celle qui, venue de la vallée du Nil, pourrait rivaliser avec des produits babyloniens (XIV, 150). Il est intéressant de constater que les vêtements offerts dans les Apophoreta comportent une synthesis, autrement dit un vêtement de banquet (explicitement présenté comme étant celui qu'on porte lorsque la toge est au repos: XIV, 142): or, la synthesis pourrait passer pour un emblème de la poésie de Martial dont les Apophoreta s'ouvrent précisément sur le mot de synthesibus ${ }^{92}$. Des étoffes légères (leuia) sont qualifiées de peu utiles par temps hivernal et sont opposées à la laine (du poète, comme le suggère l'emploi du déterminant possessif ?) - uilli mei - qui apporte, elle, de la chaleur : la leuitas des tissus (et des textes) siérait donc moins au temps des Saturnales, que la grosse laine (et les plaisanteries épaisses) de Martial. Du côté de la simplicité, on trouve aussi des manteaux de Bétique (XIV, 133) qui pourraient constituer une forme d'emblème de la poésie de Martial : ces manteaux se vantent en effet de ne pas posséder une teinte menteuse (mendax), contrairement aux vêtements qui captivent l'œil par leur pourpre tyrienne. Il me paraît intéressant de trouver, jointe à l'image d'une étoffe d'origine ibérique, l'idée d'une simplicité, d'une fidélité au réel, et d'un refus des cosmétiques et des teintures d'emprunt ${ }^{93}$.

Autre produit d'origine ibérique : le poignard (XIV, 33) dont la lame a été refroidie dans les eaux fraîches du Salo, fleuve qui arrose Bilbilis. Là encore, il est intéressant de trouver, chez un poète friand de l'acute dictum, l'image du poignard que Cicéron associait à la breuitas et à la rapidité du rythme (Orator, 224) et de constater que Martial s'attache précisément au moment où la lame est plongée dans l'eau fraîche d'un fleuve, concentrant ainsi en une brève vignette qui comprend les mots breuis et uena (qui désigne à la fois la veine poétique et le membre viril) et nous dépeint l'image d'une sphragis (orbita) apposée au poignard. Ces images potentiellement métapoétiques sont par ailleurs évoquées par des termes (stridentem tinxit) qui rappellent, me semble-t-il, la description virgilienne de la forge d'où sortira le bouclier d'Énée (stridentia tingunt : Én. VIII, 450).

Une éventuelle signature de Martial se retrouve aussi dans le cycle d'épigrammes relatives aux vins, à la fin du recueil des Xenia : le poète y associe en effet, dans deux poèmes successifs (128-129), une description du vin de Tarragone, vin ibérique, et d'un vin de Nomentum, explicitement présenté comme étant la récolte personnelle du poète (meum... Bacchum) qui y possédait une terre. Autre exemple qui nous intéresse de près : le vin pélignien, qui rappelle les origines d'Ovide - le Paelignus poeta -, est présenté comme turbata, et bon pour la consommation d'un affranchi mais non du destinataire de l'épigramme de Martial. S'agirait-il d'un clin-d'œil critique à l'endroit d'Ovide?

91 Les ailes de Dédale, autre symbole métapoétique d'origine ovidienne et que Martial associe en plusieurs lieux à Ovide, sont elles aussi volontiers citées par Martial dans ses autres livres : Spect. 10... Voir Hinds.

92 Pour cette remarque, voir Barchiesi A., art. cit., p. 327. Hinds S., art. cit., p. 140-141 et n. 84 (où l'auteur commente notamment le statut de la synthesis comme « anti-toge » dans l'œuvre de Martial).

Martial, Ép., VII, 25, 1-2 critiquera plus tard les épigrammes d'un mauvais poète qualifiées de dulcia (« douces ») et cerussata candidiora cute (« plus blanches qu'une peau enduite de céruse »). 
Dans les épigrammes 23 et 24 des Xenia on rencontre des objets qui ne semblent pas encore avoir une portée métaphorique ou un sens métapoétique, mais qui seront relus, en ce sens, dans une épigramme ultérieure de Martial. Ces deux épigrammes se présentent en effet comme un couple de contraires, la douceur des coings mélimèles (XIV, 24) étant opposée au piquant et à l'âcreté de la figue de Chios (XIII, 23). Cette opposition binaire, assez typique des couples d'objets que Martial constitue au fil des Xenia et, plus nettement encore, dans les Apophoreta, sera reprise pour décrire les qualités opposées du style de Martial et de l'un de ses rivaux dont il daube les épigrammes « douces » et efféminées à force d'êtres fardées à la céruse (VII, 25). Or, dans ce texte programmatique, Martial opposera le doucereux et la fadeur des épigrammes de son rival qui convient au goût des enfants à sa propre préférence qui va au piquant des figues de Chios. On perçoit ici à quel point les premiers recueils de Martial serviront en quelque sorte de réservoirs d'images pour ses épigrammes ultérieures ${ }^{94}$.

Pour conclure ces quelques réflexions sur le regard que Martial porte sur ses modèles, j'aimerais revenir sur les célèbres recusationes où l'épigrammatiste annonce son refus de la poésie mythologique (Ép., IV, 49 et X, 4) et prononce sa célèbre formule : " hominem pagina nostra sapit » $(\mathrm{X}, 4,10)$. Renvoyant le lecteur avide de récits mythologiques à la lecture des Aitia de Callimaque plutôt qu'à celle de ses épigrammes, Martial cite une série de personnages qui ne sont pas véritablement associés, si nous nous fions du moins aux fragments qui nous sont parvenus, aux Aitia, mais plutôt à différentes épopées latines: Parthenopaeus évoque l'œuvre de Stace, Hylas évoque les Argonautiques et donc probablement Valérius Flaccus. Quant à la figure de Scylla et au distique liant l'image d'Icare et celle d'Hermaphrodite, ils dirigent immanquablement le lecteur vers les Métamorphoses d'Ovide. Dans l'épigramme X, 4, Martial centre son attention sur le rapport que les poèmes mythologiques entretiennent avec le réel. Bien que ce texte soit largement postérieur aux Xenia et aux Apophoreta, il nous fournit sans doute une clé pour comprendre les raisons qui poussent Martial à citer Ovide dans ses premiers livres. Dans le Livre des Spectacles, Martial revient en effet sur l'idée que la réalité des spectacles mythologiques mis en scène dans le Colisée pour mettre à mort des condamnés grimés en Pasiphaé ou en Orphée dépasse les fictions des poètes, et en particulier celles des Métamorphoses d'Ovide auxquelles il fait allusion à plusieurs reprises ${ }^{95}$. Dans les Apophoreta, il décrit l'inutilité des cosmétiques, et leur caractère inadapté, étant donnée la réalité des personnages aux corps hideux et répugnants qui vont chercher à s'en servir. On peut donc supposer que ce que Martial vise ici n'est autre que le rapport d'Ovide au monde des images et à la question de la représentation. Cette idée est confortée par le choix même de décrire des objets, des xenia et apophoreta, termes qui peuvent évoquer, en particulier pour les xenia, le genre pictural de la nature morte. Or, comme l'a bien

\footnotetext{
94

95

Sur ce point, voir supra n. ??.

Sur ce type de mises en scène mythologiques, voir Coleman K., "Fatal Charades : Roman Executions Staged as Mythological Enactments », The Journal of Roman Studies, $n^{\circ}$ 80, 1990, p. 44-73, spéc. p. 62-63 sur les épigrammes Lib. Spect. 5 (« Pasiphaé »), 8 («Dédale »), 21 ( Orphée »). Par leurs thèmes et par leurs fomulations, ces épigrammes multiplient les allusions à Ovide, et notamment aux Métamorphoses. Voir par exemple Zingerle A., op. cit., p. 15, 19 et 33, qui reconnaît, dans Lib. Spect. 21, le souvenir d'Ovide, Mét., XI, 22 ; Am. I, 10, 26 et III, 5, 4. Sur le sens de ces réminiscences ovidiennes, voir Coleman K., op. cit., p. 176 (sur Lib. Spect. 21) , p. 207 (sur Lib. Spect. 28 (« Léandre ») et 247 (sur Lib. Spect. 33 (biche évoquant l'Actéon des Métamorphoses)) et Hinds S., 2007, art. cit., p. 148-151.
} 
montré $\mathrm{N}$. Bryson ${ }^{96}$, l'emploi de ce genre dans la peinture est associé, de manière privilégiée, à une réflexion ambitieuse sur le problème de l'illusion et du rapport au réel et le motif de nature morte va bien souvent être celui qui devra inciter le spectateur d'un décor à réfléchir sur les degrés de représentation impliqués par l'image, sur le seuil entre monde des images et monde réel. Si Martial cite donc à plusieurs reprises un Ovide qui peut-être perçu comme " prémartialien » ou comme un auteur de quasi-épigrammes insérées dans une trame mythologique, il cherche aussi à prendre ses distances face à une forme d'alexandrinisme perçue comme éloignée de la réalité et efféminée ou caractérisée par une afféterie excessive.

N. Bryson, op. cit., chap. 1. Soulignons cependant que les peintures à motifs de xenia qui induisent une réflexion particulièrement ambitieuse sur les seuils entre monde des images et monde réel sont celles identifiées dans les décors de deuxième style pompéien, autrement dit dans des décors élaborés à l'époque républicaine. Cette dimension demeure cependant présente dans la réception ultérieure des motifs de xenia comme le suggère par exemple l'importance conférée à ce motif par Philostrate l'Ancien dans ses Eikones. 
- Ambühl, A. (2005) : Kinder und junge Helden. Innovative Aspekte des Umgangs mit der literarischen Tradition bei Kallimachos, Louvain, Peeters.

- Barchiesi, A. (2005) : "The Search for the Perfect Book : A PS to the New Posidippus ", dans K. Gutzwiller (dir.), The New Posidippus. A Hellenistic Poetry Book, Oxford, Oxford University Press, p. 320-342.

- Bryson, N. (1990) : Looking at the Overlooked. Four Essays on Still Life Painting, Londres, Reaktion books.

- Citroni, M. (1989) : « Marziale e la letteratura per i Saturnali (poetica dell' intrattenimento e cronologia della pubblicazione dei libri) », Illinois Classical Studies 14, p. 201-226.

- Citroni, M. (2005) : "Finalità e struttura della rassegna degli scrittori greci e latini in Quintiliano », dans F. Gasti - G. Mazzoli (dir.), Modelli letterari e ideologia nell'età flavia. Atti della III Giornata ghisleriana di Filologia classica (Pavia, 30-31 ottobre 2003), Côme - Pavie, p. 15-38.

- Coleman, K. (1990) : «Fatal Charades: Roman Executions Staged as Mythological Enactments », The Journal of Roman Studies, 80, p. 44-73.

- Coleman, K. (2006): Martial : Liber Spectaculorum. With introduction, translation and commentary, Oxford : Oxford University Press.

- Connors, C. (1992) : « Seeing Cypresses in Virgil », The Classical Journal 88-1, p. 1-17.

- Faber, R. (1995) : «Vergil Eclogue 3.37, Theocritus 1 and Hellenistic Ekphrasis », American Journal of Philology 116, p. 411-417.

- Faber, R. (2000) : " The Literary Metaphor of the Chisel (Tornus) in Eclogue 3.38 », Hermes 128, p. 375-379.

- Faber, R. (2008) : « The Woven Garment as Literary Metaphor : The Peplos in Ciris 9-41 », dans J. Edmonson - A. Keith (dir.), Roman Dress and the Fabrics of Roman Culture, Toronto, University of Toronto Press, p. 205-216.

- Grewing, F. (2002) : compte rendu de T. J. Leary, Martial Book XIII : The Xenia. Text with introduction and commentary, Londres, Duckworth, 2001, Bryn Mawr Classical Review, 2002.08.38 :

http://bmcr.brynmawr.edu/2002/2002-08-38.html

- Heldmann, K. (1982) : « Ovid Über den Pfau : Zum Lobe der Schönheit », Hermes 110, p. 375380.

- Henriksén Chr. (2004) : compte rendu de C. Schöffel, Martial, Buch 8. Einleitung, Text, Übersetzung, Kommentar, Stuttgart, Franz Steiner, 2002, Bryn Mawr Classical Review, 2004.01.21 :

http://bmcr.brynmawr.edu/2004/2004-01-21.html

- Hinds, S. (2007) : « Martial's Ovid / Ovid's Martial », The Journal of Roman Studies 97, p. 113154.

- Holzberg, N. (2002) : Martial und das antike Epigramm, Darmstadt.

- Jackson, S. (2001) : «Callimachus : Coma Berenices : Origins », Mnemosyne, 4e série, 54-1, p. 1-9.

- Klein, F. (2008) : La leuitas dans l'œuvre ovidienne. Étude d'une catégorie poétique dans le système littéraire de la Rome augustéenne, thèse de doctorat de l'Université Lille 3 - Charles-deGaulle.

- Leary, T. J. (1996) : Martial Book XIV: The Apophoreta. Text with introduction and commentary, Londres, Duckworth.

- Leary, T. J. (1997) : « Martial 14.100 : Panaca », The Classical Quarterly 47, p. 322-323. 
- Leary, T. J. (2001) : Martial Book XIII : The Xenia. Text with introduction and commentary, Londres, Duckworth.

- Laurens, P. (1992) : « La poétique de la langue ou la performance descriptive dans le livre VI de l'Anthologie grecque et dans les livres XIII et XIV de Martial », Revue de Philologie 66-2, p. 301-315.

- Lehmann, K. (1945) : «A Roman Poet Visits a Museum », Hesperia. Journal of the American School of Classical Studies at Athens 14, p. 259-269.

- Mattiacci, S. (2007) : "Marziale e il neoterismo », dans A. Bonadeo - E. Romano (dir.), Dialogando con il passato. Permanenze e innovazioni nella cultura latina di età flavia, Florence, p. 177-206.

- Pitcher R. A. (1998) : "Martial's debt to Ovid », dans F. Grewing (dir.), Toto Notus in Orbe : Perspektiven der Martial-Interpretation, Stuttgart, Franz Steiner Verlag, p. 59-76.

- Poliakoff M. (2008): «Nectar, Springs, and the Sea : Critical Terminology in Pindar and Callimachus », ZPE 39, p. 41-47.

- Preston, K. (1920) : «Martial and Formal Literary Criticism », Classical Philology 15, p. 340352.

- Prioux, É. (2008) : Petits musées en vers. Épigramme et discours sur les collections antiques, Paris, CTHS/INHA.

- Prioux, É. (2009a) : « Ovide, De Medicamine faciei », dans I. Bardiès-Fronty - Ph. Walter - M. Bimbenet Privat (dir.), Le Bain et le Miroir. Catalogue de l'exposition du Musée National du Moyen Âge et du Musée National de la Renaissance (mai-septembre 2009), Paris, Gallimard, p. 231-233.

- Prioux, É. (2009) : «On the Oddities and Wonders of Italy: When Hellenistic Poets Look Westward ", dans M. A. Harder, R. Regtuit, G. C. Wakker (dir.), Nature and Science in Hellenistic Poetry, Louvain, Peeters, p. 121-148.

- Roman, L. (2001): "The Representation of Literary Materiality in Martial's Epigrams », Journal of Roman Studies 91, p. 113-145.

- Roman, L. (2006) : « A History of Lost Tablets », Classical Antiquity 25-2, p. 351-388.

- Rosati, G. (1985) : Ovidio. I cosmetici delle donne, Venise, Marsilio.

- Salemme, C. (1976) : Marziale e la «poetica» degli oggetti : struttura dell'epigramma di Marziale, Naples, Società editrice napoletana.

- Salemme, C. (2005) : Marziale e la poesia delle cose, Naples, Loffredo.

- Szelest, H. (1999) : «Ovid und Martial », dans W. Schubert (dir.), Ovid : Werk und Wirkung . Festgabe für Michael von Albrecht zum 65. Geburtstag, Frankfurt-am-Main, Peter Lang, t. 2, p. 861-864.

- Siedschlag, E. (1972) : « Ovidisches bei Martial », Rivista di Filologia e di Istruzione Classica 100, p. 156-161.

- Spaeth Jr., J. W. (1930) : «Martial and Vergil », Transactions and Proceedings of the American Philological Association 61, p. 19-28.

- Sullivan, J. P. (1991) : Martial, the Unexpected Classic: A Literary and Historical Study, Cambridge, Cambridge University Press.

- Watson, P. A. (1982) : "Ovid and Cultus : Ars Amatoria 3.113-328 », Transactions of the American Philological Association, 112, p. 237-244.

- Watson, P. A. (2001) : "Parody and Subversion in Ovid's Medicamina faciei femineae », Mnemosyne, $4^{\mathrm{e}}$ série, 54, p. 457-471. 
- Worman N. (2009) : « Bodies and Topographies in Ancient Stylistic Theory », dans Th. Fögen - M. M. Lee (dir.), Bodies and Boundaries in Graeco-Roman Antiquity, Berlin - New York, Walter de Gruyter, p. 45-62.

- Young Forsyth, P. (1985), « Gifts and Giving : Catullus 12-14 », The Classical World 78-6, p. 571-574.

- Zingerle A. (1877), Martial's Ovid-Studien, Innsbruck, Wagner'sche UniversitätsBuchhandlung.

- Zissos, A. (2004) : «Navigating Genres: Martial 7.19 and the Argonautica of Valerius Flaccus », The Classical Journal 99-4, p. 405-422. 